\title{
SENSORIAMENTO REMOTO APLICADO AOS ESTUDOS GEOGRÁFICOS: CONSIDERAÇÕES A PARTIR DOS TRABALHOS SUBMETIDOS AO XI ENANPEGE
}

\author{
Applied remote sensing in geography: considerations \\ from works submitted to XI Enanpege
}

Teledetection appliquee aux etudes geographiques: les considerations des communications presentees au XI Enanpege

\section{Ericson Hideki Hayakawa}

Doutorado em Sensoriamento Remoto pelo Instituto Nacional de Pesquisas Espaciais. Docente no Programa de Pós-graduação em Geografia da Universidade Estadual do Oeste do Paraná-Campus de Marechal Cândido Rondon. Universidade Estadual do Oeste do Paraná - Unioeste. Rua Pernambuco, 1777 - Centro - Marechal Cândido Rondon - CEP 85960000.

E-mail: ericson.geo@gmail.com

\section{Vitor Matheus Bacani}

Doutorado em Geografia Física pela Universidade de São Paulo (USP/FFLCH). Docente nos Programas de Pós-graduação em Geografia da Universidade Federal de Mato Grosso do Sul (Campi de Aquidauana e Três Lagoas) e do Programa de Pós-Graduação em Recursos Naturais da UFMS/FAENG em Campo Grande. Universidade Federal de Mato Grosso do Sul, Campus de Três Lagoas, Unidade 2. Av. Ranulpho Marques Leal, 3484 / CEP 79620-080. Três Lagoas-MS.

E-mail:vitormbacani@gmail.com

\section{Fábio Marcelo Breunig}

Doutorado em Sensoriamento Remoto pelo Instituto Nacional de Pesquisas Espaciais. Docente na Universidade Federal de Santa Maria, junto ao Departamento de Engenharia Florestal e do Programa de Pós-graduação em Geografia. Universidade Federal de Santa Maria (UFSM). Linha Sete de Setembro $s / n$ - BR386 KM40. Sala 59 Bloco de apoio 4. CEP: 98400-000 - Frederico Westphalen - RS

E-mail:fabiobreunig@gmail.com

\section{Aguinaldo Silva}

Doutorado em Geociências e Meio Ambiente pela Universidade Estadual Paulista Júlio de

Mesquita Filho. Docente nos Programas de Pós-graduação em Geografia e Estudos Fronteiriços da Universidade Federal de Mato Grosso do Sul (UFMS). Universidade Federal de Mato Grosso do Sul Campus do Pantanal, Avenida Rio Branco, 1270 - Bairro Universitário - 79.304-902 - Corumbá-MS; E.mail:aguinald_silva@yahoo.com.br

\section{Romário Trentin}

Doutor em Geografia pela Universidade Federal do Paraná. Docente do Programa de Pós-Graduação em Geografia da Universidade Federal de Santa Maria. Universidade Federal de Santa Maria - Campus da UFSM - Av. Roraima, 1000 - Prédio 17, sala 1113c-Bairro Camobi - CEP:97105-900 - Santa Maria/RS. E-mail: romario.trentin@gmail.com 


\section{Waterloo Pereira Filho}

Doutorado em Geografia pela Universidade de São Paulo e pós-doutorado pela University of Victoria - Canadá. Docente na UFSM - Universidade Federal de Santa Maria. Universidade Federal de Santa Maria / UFSM, Sala 2048, Avenida Roraima, 1000. Bairro: Camobi Cidade Universitária. CEP: 97105-970, Santa Maria, RS. Caixa Postal: 5021.

E-mail:waterloopf@gmail.com

\section{RESUMO}

O Sensoriamento Remoto (SR) é um dos principais representantes das geotecnologias. A constante inovação no SR e nas geotecnologias exigem cuidados no tocante aos procedimentos metodológicos, a fim de assegurar resultados confiáveis. O objetivo deste trabalho é verificar as principais aplicações do SR na geografia, e discutir sobre os entraves e potencialidades verificados pela comunidade geográfica, a partir da análise dos trabalhos submetidos no Grupo de Trabalho: Sensoriamento Remoto aplicado aos Estudos Geográficos, do ENANPEGE, 2015. Nota-se a expressiva utilização do SR em estudos da geografia. Contudo, ao contrário do patamar de desenvolvimento que se encontram as ferramentas e técnicas de SR (ex. diversidade de sensores, resoluções, etc.), parte das aplicações presentes nos trabalhos indica a subutilização do SR. Embora existam demandas de estudos que se pautem no uso do SR e demais geotecnologias na geografia, ainda é necessário investir na formação teórica e prática dos usuários.

Palavras-chave: geotecnologias, geografia, procedimentos metodológicos.

\section{ABSTRACT}

Remote Sensing (RS) is one of the most important examples of geotechnology. Constant innovation in RS and in the geotechnology require care regarding the procedures in order to ensure reliable results. The aim of this study is to verify the main applications of RS in geography, and discuss about the obstacles and possibilities verified by geographic community, from the analysis of the papers submitted to the Working Group: Remote Sensing applied to the Geographical Studies, in the ENANPEGE, 2015. Note the expressive use of RS in geography applications. However, unlike the high development stage of the RS tools and techniques (ex. several sensors, resolutions, etc.), part of the $\mathrm{RS}$ application is underused. While there are demands for studies that are guided from the use of RS and other geotechnology, it is still necessary to invest in theoretical and practical training of users.

Keywords: geotechnology, geography, procedures.

\section{RESUMÉ}

La télédétection s'est consolidé comme une des principaux représentants de la géomatique. L'innovation constante dans la télédétection et la géomatique exigent une attention en ce qui concerne les procédures méthodologiques afin d'assurer des résultats fiables. Le but de cette étude est de vérifier les principales applications de la télédétection dans la géographie, et discuter des principaux obstacles et les potentialités vérifiés par communauté géographique, à partir de l'analyse des articles soumis au Groupe de 
Travail: La Télédétection Appliquée aux Etudes Géographiques, dans l'ENANPEGE 2015. Il a été noté l'utilisation expressive de la télédétection dans les applications en géographie. Cependant, contrairement à la phase de développement qui sont les outils et les techniques de la télédétection (ex. la diversité des résolutions, capteurs, etc.) de l'application présente dans le travail de la télédétection indique la sous-utilisation. Bien qu'il existe des exigences des études qui sont basés en l'utilisation de la télédétection et d'autres géotechnologies en géographie, il est encore nécessaire d'investir dans la formation théorique et pratique des utilisateurs..

Mots-clés: Géomatique, géographie, procédures méthodologiques.

\section{INTRODUÇÃO}

As geotecnologias têm despontado como uma das áreas que mais se desenvolverão (GEWIN, 2004). Na geografia, as geotecnologias são utilizadas em diferentes temáticas e aplicações (Rosa, 2005; Florenzano, 2005). Nesse contexto, o sensoriamento remoto (SR) consolidou-se no escopo das geotecnologias, e está cada vez mais presente no cotidiano dos cidadãos. Diariamente nos diferentes meios de comunicação (televisão, jornais, revistas, livros, dentre outros), inúmeras dinâmicas espaciais são exemplificadas com o apoio de imagens de satélites. No cenário acadêmico, diferentes áreas do conhecimento utilizam do SR como parte essencial ou complementar para o desenvolvimento das pesquisas. Nos últimos anos, com o lançamento de inúmeros instrumentos imageadores a bordo de satélites, em aeronaves tripuladas ou nos veículos aéreos não tripulados (VANTs) e, mais recentemente, em drones, ampliaram o leque de obtenção de imagens e demais dados, e consequentemente, das aplicações do SR. Se de um lado, as diferentes resoluções espaciais, espectrais, radiométricas e temporais dos novos sensores remotos otimizaram e potencializaram a aplicação do SR, de outro lado, também problematizaram, principalmente nas discussões que envolvem a correta utilização da técnicas e métodos do SR. Estas reflexões são fundamentais para assegurar a precisão e acurácia dos resultados e garantir a confiabilidade das soluções geradas.

Na Geografia, a utilização do SR gradativamente tem-se tornado expressiva. Observa-se o aumento no número de pesquisas e a consolidação de grupos de pesquisas que se pautam na utilização do sensoriamento remoto em aplicações geográficas. Neste contexto, mesmo que as aplicações do SR comumente estejam associados a outras ferramentas que integram as geotecnologias, como os Sistemas de Informação Geográfica (SIG) e o geoprocessamento, viu-se a possibilidade de ampliar as discussões sobre o SR no XI ENANPEGE, realizado em outubro de 2015, na cidade de Presidente Prudente, Brasil. 
A possibilidade de discussão do SR dá-se não só em função da sua amplitude de aplicações, mas também da complexidade que pode envolver as pesquisas neste tema. Por exemplo, previamente as suas aplicações, o SR exige o conhecimento dos princípios físicos que o regem, bem como, de suas possibilidades e limitações. As diferentes resoluções espaciais, espectrais, radiométricas e temporais, a forma de obtenção de dados, dentre outros, definem particularidades de aplicações. Em termos de aplicações, podemos citar aquelas elencadas na obra de Jensen (2009): SR no infravermelho termal, SR por microondas ativas e passivas, SR por LIDAR, SR da vegetação, SR da água, SR da paisagem urbana, SR de solos, minerais e geomorfologia. Em cada uma dessas aplicações, diversas particularidades devem ser respeitadas. Ademais, as pesquisas que usam o SR aplicado, a complexidade tem avançado bastante. Citam-se ainda, demais conhecimentos inerentes, como interpretação de imagens e a resposta espectral de alvos, processamento digital de imagens, fotogrametria, dentre outros.

Diante deste cenário, o GT teve por objetivo avaliar as principais aplicações do sensoriamento remoto na geografia, pautando-se nos trabalhos submetidos. Adicionalmente, procurou discutir os principais entraves e potencialidades verificados pela comunidade geográfica. Essas discussões são essenciais para conhecer alguns dos trabalhos do SR na geografia, e indicar as possíveis alternativas para assegurar maiores investimentos nessa área do conhecimento, contribuindo para a formação sólida do geógrafo e do professor de geografia no Brasil. Também procedeu-se com a análise dos trabalhos apresentados no XVI Simpósio Brasileiro de Geografia Física Aplicada (SBGFA).

\section{SENSORIAMENTO REMOTO E A GEOGRAFIA}

A definição de SR pode ser encontrada com algumas variações na literatura. Segundo Lillesend et al. (2008) e Jensen (2009), é a ciência e/ou arte de obter informações sobre um objeto, área ou fenômeno a partir de um equipamento sem estar em contato físico direto com o alvo investigado. Ciência porque engloba diferentes ramos de conhecimento (ciências exatas, biológicas, sociais, dentre outras), fundamentado em regras e leis. Arte, porque além de contemplar o conhecimento científico, representa o conhecimento empírico do indivíduo. Esta definição é próxima a verificada no Manual de Sensoriamento Remoto (Simonetti; Ulaby, 1983). Mather (1999) propõe que o SR do ambiente é a obtenção de medidas a distância, a partir das características espectrais da superfície terrestre e da atmosfera.

Para alguns autores, a origem do SR está ligada ao desenvolvimento dos sensores fotográficos (American Society of Photogrammetry - ASP, 1975). Segundo consta no Manual de Sensoriamento Remoto - Manual of Remote Sensing (ASP, 1975), a história 
do SR pode ser dividido em dois períodos principais: o período de 1860 a 1960, no qual o SR era baseado na utilização de fotografias aéreas e, o período de 1960 até os dias atuais, caracterizado pela multiplicidade de sensores orbitais (NOVO, 1988).

Várias são as formas de se caracterizar os sistemas de SR. Por exemplo, podem ser discriminados, quanto ao nível de aquisição (terrestre, a exemplo de laboratório e em campo, aéreo e orbital), quanto a fonte de energia radiante (ativos, a exemplo dos radares imageadores, os quais emitem sua própria energia, ou passivos, a exemplos dos sensores ópticos, que dependem da radiação eletromagnética proveniente principalmente do sol, ou da terra, no caso da faixa do termal), quanto ao tipo de produto gerado ou formato do dado produzido (imageadores, na forma de imagens raster, e não imageadores, na forma de gráficos, tabelas), quanto a região do espectro que operam (visível, infravermelho próximo, infravermelho médio, infravermelho distante ou termal, e microondas), dentre outras características. Em suma, o SR pauta-se nos seguintes componentes (Figura 1): a) Fonte de energia ou iluminação; b) propagação da radiação eletromagnética (REM) e atmosfera; c) Interação com os alvos/objetos da superfície terrestre; d) retransmissão da REM na atmosfera; e) registro da energia pelos sensores instalados em diferentes estruturas; f) geração dos produtos de SR que foram transmitidos e processados em terra; g) Interpretação e análise dos dados de SR; h) geração de produtos e informações, e; i) usuários de diferentes áreas do conhecimento.

Figura 1 - Representação resumida dos elementos envolvidos no SR de alvos da superfície terrestre. (Adaptado de Lillesand, et al., 2008).

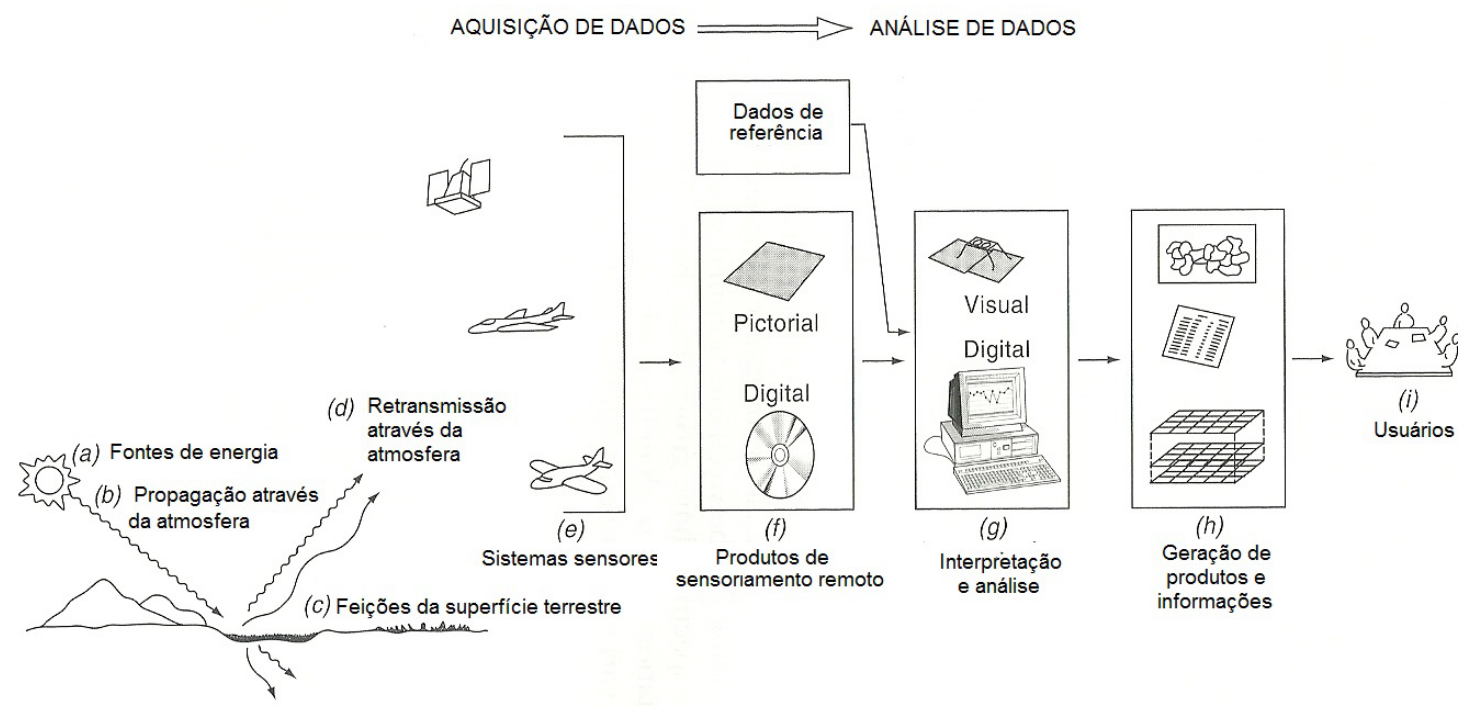


Na geografia há inúmeras possibilidades de aplicações de SR. Propriedades do SR como diferentes resoluções temporais e espaciais das imagens de satélite, são um dos atrativos para a sua utilização em uma série de estudos que contemplem fatos e fenômenos de interesse da geografia. Contudo, os dados de SR muitas vezes são subutilizados, e não se explora o seu real potencial, tanto nos aspectos qualitativos, como quantitativos (Montanher e Souza Filho, 2011). Esta conjuntura segundo Florenzano (2005), ocorre em grande parte, pela formação inicial deficiente e da ausência de formação continuada nesta área do conhecimento, o que impede a atualização profissional em uma área onde os avanços tecnológicos são constantes.

Os dados de SR são analisados comumente a partir do Processamento Analógico (interpretação visual) de Imagens e, pelo Processamento Digital de Imagens (Jensen, 2009 e Montanher, 2016). Se no passado, o uso pioneiro de fotografias aéreas e mais recentemente, das imagens de satélite pautavam-se quase que exclusivamente na fotointerpretação ou na análise qualitativa, atualmente há inúmeros outros recursos. A disponibilidade de recursos computacionais e de aplicativos de processamento digital de imagens (PDI) permite explorar os dados de SR sob outras perspectivas, especialmente, a quantitativa.

$\mathrm{Na}$ abordagem fotointerpretativa, Lillesand et al. (2008) e Jensen (2009), apresentam ampla documentação sobre os encaminhamentos teóricos e práticos para as aplicações pautadas na fotointerpretação de imagens de satélite. De modo geral, os elementos utilizados na interpretação de imagens são: a escala de cinza, o tom, a cor, a altura (profundidade), o tamanho, a forma, a sombra, a textura, a localização, a associação e o arranjo (Jensen, 2009). Conforme o autor supracitado, nesta forma de análise, há empecilhos como, a limitada capacidade do ser humano em distinguir níveis de cinza (comumente na imagem há mais informações que o intérprete consiga identificar), a inserção da subjetividade de cada intérprete, dentre outros. De outro lado, o ser humano tem competência para agregar o conhecimento prévio a outras informações, o que potencializa a interpretação. Também é possível obter medições a partir dos dados de SR, como comprimento, área, perímetro, volume, dentre outros, ou utilizar de técnicas fotogramétricas (Jensen, 2009).

Dependendo do objetivo, os resultados de aplicações pautadas na interpretação visual de dados de SR podem ser superficiais e genéricos, conforme exemplo citado em Ponzoni et al. (2012). A análise comumente pode ser limitada a uma única imagem por vez, ou integrando cenas multiespectrais em composições coloridas. De modo geral, as análises por interpretação visual não garantem resultados satisfatórios ou estatisticamente confiáveis ou validáveis. Contudo, mesmo na abordagem fotointerpretativa ou qualitativa, a formação mínima sobre os fundamentos de SR são necessários. O conhecimento de elementos como: 
a forma de coleta de dados, os princípios de radiação eletromagnética, o comportamento espectral de alvos, a interação entre energia e matéria no terreno e na atmosfera, o período de aquisição dos dados, as características dos sensores, dentre outras particularidades, contribuem para interpretações coerentes.

O Processamento Digital de Imagens ou abordagem quantitativa, conforme Jensen (2009), também faz o uso de elementos de interpretação de imagens. Contudo, com o apoio de recurso computacional. A própria condição atual do SR, e a sua diversidade de dados em diferentes resoluções temporais, espectrais, temporais e radiométricas, comumente mais detalhados, exigem formas robustas de análise de dados. Por isso a necessidade de potencializar o PDI, a fim de obter dados de caráter quantitativo, e biofísicos (Jensen, 2005). No contexto quantitativo, comumente há a conversão de valores físicos considerando as particularidades de cada faixa espectral: reflectância para a faixa espectral do visível, infravermelho próximo e de ondas curtas; temperatura e brilho para a faixa espectral do termal; e o coeficiente de retroespalhamento para a faixa espectral das micro-ondas (Xingfa et al., 2005). Os dados geofísicos e biofísicos referem-se à obtenção de informações sobre a temperatura da superfície terrestre, a biomassa, a precipitação, os índices de área foliar, a temperatura superficial dos oceanos, a evapotranspiração, dentre outros (Jensen, 2005; Xingfa et al., 2005).

$\mathrm{Na}$ abordagem quantitativa, deter certo grau de habilidade matemática e estatística, adicionada a alguma familiaridade com os recursos computacionais (Mather, 1999) é positiva e potencializa as pesquisas. Segundo o autor supracitado, o especialista de SR, na realidade, não atua apenas nesse tema, mas deve ter o conhecimento de inúmeras outras áreas do conhecimento. Os exemplos básicos de procedimentos de PDI envolvem correções radiométricas e geométricas, realce de imagens, reconhecimento de padrões a partir de estatística inferencial, sistemas especialistas (árvores de decisão), análise de imagens a partir de redes neurais, análises de dados hiperespectrais, detecção de mudanças (Jensen, 2005). Adicionalmente, quando os dados de SR são utilizados para fins cartográficos, o que no passado era realizado por interpretação convencional, atualmente se recorre a procedimentos de PDI (Freitas, 2014).

A despeito da forma analógica e/ou digital de utilização do SR, destacam-se as reflexões que incidem sobre esta área do conhecimento, e das geotecnologias como um todo, e seus desdobramentos na geografia. Machado (2014) reflete sobre o desenvolvimento recente e constante das Tecnologias da Informação Geográfica. O autor supracitado chama a atenção para a atual conjuntura na geografia, que indica uma mudança de paradigma, que conduzirá através do estabelecimento de fundamentos teóricos, conceituais e ontológicos, 
à consolidação da Ciência da Informação Geográfica. O autor indica que este processo beneficiará várias áreas do conhecimento geográfico, independente dos aspectos físicos e humanos. Discussões neste sentido são positivas, e felizmente tem surgido na geografia.

Neste contexto, destaca-se a obra de Estes e colaboradores (Estes et al., 1980), sobre os Impactos do Sensoriamento Remoto na Geografia dos Estados Unidos ("Impacts of Remote Sensing on U.S. Geography"). Naquele momento, o SR ainda era uma tecnologia relativamente nova, mas que gradativamente consolidava-se, especialmente na geografia (quando comparada a outras áreas do conhecimento). Os autores estavam preocupados sobre os impactos do SR nas pesquisas geográficas. Questionavam sobre: i) o que o geógrafo poderia realizar de forma melhor e com menos custos financeiros que no passado a partir do uso de dados de SR; ii) o que o SR permite ao geógrafo realizar hoje e que não conseguia realizar no passado e; iii) o que o geógrafo do futuro poderia realizar com o SR que seria significante, tanto profissionalmente como academicamente. Estes et al. (1980) teciam demais ponderações como: i) a presença de poucos pesquisadores que utilizam do SR nas pesquisas; ii) alguns geógrafos econômicos perceberam o potencial do sensoriamento remoto; iii) o interesse de geógrafos regionais pelo uso do SR em suas pesquisas, e o interesse de países em desenvolvimento pela nova tecnologia e; iv) o aumento de cursos relacionados ao SR ofertados pelos departamentos de geografia, mas que não teria gerado a elevação no número de publicações sobre SR em periódicos científicos.

É notável como vários dos questionamentos e demais ponderações emitidos na década de 1980 ainda são atuais. A despeito dos elementos elencados, os autores afirmavam que o sensoriamento remoto se tornou uma realidade na geografia e acrescentaram que, era uma ferramenta poderosa e que não poderia ser ignorada. Chama a atenção que, embora tardia na geografia brasileira, Estes e colaboradores já na década de 1980 previam que esta nova ferramenta mudaria as nossas percepções, os nossos métodos de análises de dados, os nossos modelos e os nossos paradigmas. Condição esta também destacada por Machado (2014), e que parece estar em curso. Atualmente, é notável a farta literatura acerca de exemplos de SR aplicado em diferentes abordagens geográficas. Pioneiramente, Estes et al. (1980), pautando-se na obra de David Harvey (Explanation in Geography), elencaram a potencial habilidade do SR em contribuir nos típicos estudos geográficos. Mais recentemente, obras como a de Florenzano (2008), Boyd (2009), Jensen (2009), Longley et al. (2013) apresentam inúmeros exemplos de potenciais estudos de cunho geográfico, pautando-se no uso do SR.

Diante deste cenário, é importante ressaltar que o Brasil é um dos países da América do Sul com destaque na área do conhecimento referente ao SR. A presença da Divisão de Sensoriamento Remoto (DSR) no Instituto Espacial de Pesquisas Espaciais (INPE), 
propiciou não só a realização de relevantes pesquisas acerca desta área do conhecimento, como também contribuído para a formação de recursos humanos, que atualmente encontram-se alocados em diferentes setores profissionais. Em concordância com Gewin (2004), no Brasil a área de geotecnologias (dentre elas o SR) tem recebido grande atenção por parte da comunidade acadêmica e junto as empresas. Em termos de formação acadêmica, os cursos de Pós-Graduação em Sensoriamento Remoto estão disponíveis no INPE e na Universidade Federal do Rio Grande do Sul (UFGRS). Contudo, ainda é pequeno o número bacharéis e licenciados em geografia que obtiveram o título de mestres e/ou doutores na área de SR. Esse fato, associado a demanda do mercado, faz com que exista uma carência de profissionais.

\section{TRABALHOS E DISCUSSÕES DECORRENTES DO GT NO XI ENANPEGE}

O GT intitulado “Sensoriamento Remoto aplicado aos estudos Geográficos” ocorreu pela primeira vez no ano de 2015, no XI ENANPEGE. Nessa edição do evento, também ocorreu o GT “Geocartografia, geoprocessamento e análise espacial”. O GT "Sensoriamento Remoto aplicado aos estudos Geográficos", contou com a apresentação de 06 trabalhos, em um total de 11 trabalhos aprovados. Embora o número de trabalhos não tenha sido expressivo, a discussão gerada no evento foi positiva. Do ponto de vista reflexivo, esta condição pode suscitar uma série de questionamentos como: i) que na geografia, a utilização do SR ainda é incipiente; ii) que os pesquisadores que utilizam do SR não tiveram conhecimento deste GT ou interesse na submissão do trabalho ao ENANPEGE; iii) que os pesquisadores que utilizam do SR submetem os trabalhos em eventos específicos, como o Simpósio Brasileiro de Sensoriamento Remoto (SBSR) ou o Congresso Brasileiro de Cartografia (CBC). Há também a possibilidade de trabalhos como as aplicações de SR no ensino de geografia estar submetidos em outros GTs, no próprio evento XI ENANPEGE.

A análise dos trabalhos consistiu principalmente na verificação dos materiais e procedimentos adotados pelos autores. Foram analisados os seguintes itens: i) quais os tipos de dados utilizados; ii) quais os aplicativos utilizados; iii) quais os procedimentos de pré-processamento realizadas: especialmente correção atmosférica e geométrica dos dados; iv) quais as técnicas de manipulação de imagens, como NDVI (Índice de Vegetação por Diferença Normalizada), segmentação, classificação, e fusão; v) presença de referencial bibliográfico em língua estrangeira.

Os resultados em relação aos tipos de dados utilizados demonstram que, as imagens utilizadas nos trabalhos são oriundas predominantemente dos sensores instalados nos satélites da série Landsat. Dos 11 trabalhos (Figura 2), 05 utilizaram de imagens do 
sensor OLI (Operational Land Imager), em concomitância com outras imagens, como do Google Earth, e Thematic Mapper (TM). Outros 05 trabalhos utilizaram de imagens TM em conjunto com dados do Aster/GDEM (Advanced Spaceborne Thermal Emission and Reflection Radiometer / Global Digital Elevation Map) e SRTM (Shuttle Radar Topography Mission), e imagens do satélite CBERS (Satélite Sino-Brasileiro de Recursos Terrestres). Um dos trabalhos utilizou imagens do satélite THEOS (Thailand Earth Observation Satellite).

Figura 2: Dados utilizados nos trabalhos submetidos ao GT. Nota-se o predomínio de imagens dos sensores OLI e TM, seguido das imagens do Google Earth, e dos demais sensores.

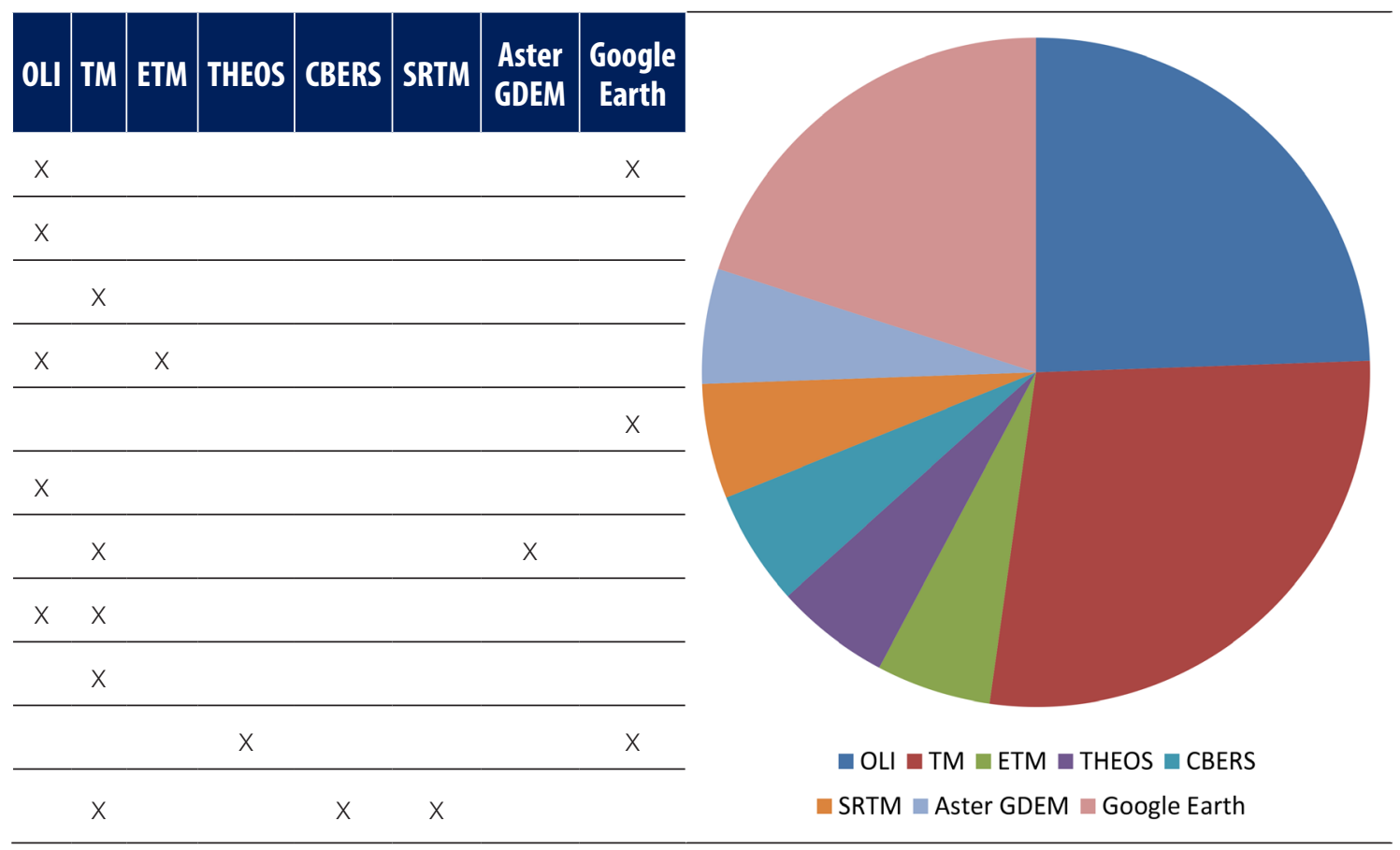

Os aplicativos comumente utilizados nos trabalhos foram respectivamente: ArcGis, SPRING, ENVI, Global Mapper e Surfer (Figura 3). Dos 11 trabalhos analisados, 09 citaram o uso do aplicativo ArcGis, 04 do aplicativo SPRING e 02 do aplicativo ENVI. Salienta-se que, alguns trabalhos realizaram o uso em paralelo de mais de um aplicativo. Em termos de pré-processamento, três trabalhos realizaram correção geométrica das imagens, e um (01) realizou também a manipulação de histograma. Quanto ao processamento dos dados de sensoriamento remoto, dos trabalhos apresentados, três utilizaram da técnica de NDVI (Índice de Vegetação por Diferença Normalizada), dois realizaram o procedimento de fusão de imagens, dois realizaram segmentação e sete trabalhos efetuaram classificação de imagens. 
Figura 3 - Principais aplicativos e técnicas utilizadas nos dados de sensoriamento remoto.

\begin{tabular}{|c|c|c|c|c|c|c|c|c|c|}
\hline SPRING & $\begin{array}{l}\text { Ano } \\
\text { GIS }\end{array}$ & ENVI & $\begin{array}{l}\text { Global } \\
\text { Mapper }\end{array}$ & Surfer & $\begin{array}{l}\text { Correção Geométrica e } \\
\text { atmosférica }\end{array}$ & NDVI & Fusão & Segmentação & Classificação \\
\hline$X$ & $x$ & & & & & & & & $x$ \\
\hline \multirow[t]{6}{*}{$x$} & $x$ & & & & & & & & \\
\hline & & & & & $x$ & & & & \\
\hline & $x$ & & & & & & & & \\
\hline & $x$ & & & & & & & & $x$ \\
\hline & $x$ & & & & & & & & \\
\hline & $x$ & $x$ & & & x (correção geométrica) & $x$ & & & \\
\hline \multirow[t]{3}{*}{$x$} & & & & & $\begin{array}{c}\text { x (correção geométrica e radiométrica } \\
\text { - manipulação de histograma) }\end{array}$ & & & & \\
\hline & $x$ & $x$ & & & & & & & \\
\hline & $x$ & & & & & & & & $x$ \\
\hline$x$ & $x$ & & $x$ & $x$ & & & & & \\
\hline
\end{tabular}

Os procedimentos realizados são aqueles já consolidados e que na maioria das vezes, encontram-se implementados nos aplicativos. Contudo, chama a atenção alguns procedimentos como: realização de fusão de imagens. Este procedimento geralmente é realizado para a obtenção de imagens de melhor resolução espacial. Entretanto, se o interesse for apenas de obter uma imagem de melhor resolução, desde o ano de 2012 é possível obter imagens para fins acadêmicos de melhor resolução espacial e radiométrica. Trata-se das imagens Rapideye, com tamanho de pixel de 5 metros e resolução radiométrica de 11 bits.

Esta possibilidade surgiu do termo celebrado no ano de 2012, entre o Ministério do Meio Ambiente (MMA), e a Empresa Santiago \& Cintra Consultoria (Contrato MMA n. 30/2012), onde a referida empresa é a distribuidora oficial de Imagens Rapideye no Brasil. Neste contrato estão presentes os termos do item 8.7, da cláusula Oitava do Contrato Administrativo n. 30/2012, o qual exige que "o tipo de licenciamento e o direito de uso das imagens deveriam permitir a distribuição de cópias aos diversos Órgãos Federais cadastrado e demais Órgãos públicos pertencentes ao Governo Federal, além de Órgãos Públicos devidamente envolvidos nos programas do Ministério do Meio Ambiente". Adicionalmente, o documento de esclarecimento enviado pela Empresa Santiago \& Cintra, afirma que " $a$ licença de uso das imagens Rapideye permite a sua utilização por todos os órgãos públicos brasileiros, em conformidade com o Contrato de Licença do Usuário Final - EULA". Neste 
contexto, a partir do preenchimento de um termo de compromisso corporativo de uso das imagens Rapideye, entre o interessado (órgão público) e a empresa Santiago \& Cintra Consultoria, é possível obter imagens deste satélite e utilizá-los nas pesquisas acadêmicas.

A utilização da técnica de NDVI também requer uma reflexão, principalmente sobre o pré-processamento das imagens de satélite. Ao utilizar a técnica de NDVI é necessária a correção atmosférica das imagens. Ponzoni et al. (2012) salientam que, para a geração das imagens índice de vegetação, é fundamental a transformação dos números digitais (NDs) das imagens para valores de Fator de Reflectância Bidirecional (FRB) de superfície. Assim é possível obter os parâmetros referentes às propriedades espectrais do alvo, no caso, a vegetação. Adicionalmente, os valores obtidos são comparáveis com os já encontrados na literatura. A não realização da conversão dos NDs para valores físicos como radiância ou FRB em aplicações envolvendo índice de vegetação poderão gerar resultados equivocados. Isto porque, os NDs das imagens não estão na mesma escala radiométrica nas diferentes bandas espectrais. A não padronização da escala radiométrica pode incorrer em equívocos quando se compara diferentes imagens e bandas espectrais. É fundamental proceder com a correção atmosférica e a conversão dos dados das imagens em valores físicos, como radiância ou reflectância de superfície em estudos que visem o cálculo do índice de vegetação. De modo geral, nota-se que há um desconhecimento ou descuido sobre o procedimento de correção atmosférica. Quando é tratado, na maioria das vezes são utilizados métodos empíricos como o da subtração do pixel escuro, devido a dificuldade em aplicar os métodos que fazem uso dos princípios de transferência radiativa.

O item referencial bibliográfico em língua estrangeira também causou surpresa. Embora nos últimos anos, o número de obras publicadas e traduzidas para o português aumentaram expressivamente, muitas das publicações referentes ao SR ainda estão na língua inglesa. Dos 11 trabalhos submetidos, apenas 02 trabalhos apresentavam citações de publicações em língua inglesa, e 01 trabalho em língua espanhola. Adicionalmente, comumente os programas de pós-graduação possuem com requisito para a seleção ou permanência no mestrado ou doutorado, a aprovação em exame de proficiência em língua estrangeira. O cumprimento deste requisito não trata apenas de mera formalidade, mas tem o intuito de que o trabalho realizado esteja sólido bibliograficamente, e tenha possibilidade de divulgação internacional, em publicações em periódicos ou eventos. Esta conjuntura deve ser analisada não somente no contexto deste GT, mas também no contexto dos próprios programas de pós-graduação. Adicionalmente, no que tange ao processo de seleção de material bibliográfico em língua estrangeira, observa-se a interdependência entre a qualidade da produção nacional e o embasamento teórico (relação de causa-efeito). 
Os trabalhos submetidos no GT referem-se principalmente a utilização de dados de sensores óticos, de média resolução espacial, de amplitude multiespectral, e que estejam disponibilizadas gratuitamente. Mesmo diante da atual diversidade de sensores e plataformas (incluindo-se aqueles instalados em VANTs e drones), as pesquisas no contexto geográfico ainda são incipientes em relação à variedade de ferramentas. Tem-se ainda um amplo leque de possíveis pesquisas, pautado no uso das ferramentas e técnicas de SR ainda pouco exploradas na geografia, como utilizar de instrumentos imageadores como radares e lasers, bem como, da exploração da potencialidade das imagens de alta resolução espacial, maior resolução radiométrica e temporal, bem como dados hiperespectrais.

Notou-se que as fontes de dados de SR utilizados na maior parte dos trabalhos eram obtidos do catálogo de imagens do INPE (Instituto Nacional de Pesquisas Espaciais), da EMBRAPA (Empresa Brasileira de Pesquisa Agropecuária), do Topodata (Bando de Dados Geomorfométricos do Brasil) e da Global Land Cover Facility. Contudo, há inúmeras outras fontes de dados, dentre as quais, destacam-se o Earth Explorer, da USGS (Serviço Geológico dos Estados Unidos). Neste é possível obter dados com maior nível de processamento, incluindo a correção atmosférica. A partir do convênio entre o Ministério de Meio Ambiente e a Empresa Santiago \& Cintra Consultoria, também é possível obter dados do satélite RapidEye, cujas imagens possuem tamanho de pixel de $5 \mathrm{~m}$.

A partir das análises dos trabalhos submetidos a este GT, nota-se a ausência de que trabalhos que envolvem a radiometria, comportamento espectral, ou a utilização de produtos oriundos de sensores instalados em radares e lasers, bem como mais recentemente, de VANTs e drones. Destaca-se também a utilização restrita de algoritmos classificadores, onde não foi identificada a utilização de abordagens relativamente comuns na literatura internacional relacionada ao SR, tais como a orientada a objeto (GEOBIA Geographic Object-Based Image Analysis) ou SVM (Support Vector Machine). Salienta-se que, partes das considerações tecidas podem ser refutadas, caso trabalhos desse caráter estejam sendo submetidos a outros eventos, como o Simpósio Brasileiro de Sensoriamento Remoto. Contudo, ainda há uma demanda para a sólida qualificação profissional em SR e geotecnologias na geografia. É fundamental a aliar a habilidade do geógrafo em analisar o espaço geográfico, muito em função da rica formação curricular, com a habilidade técnica e conceitual, por exemplo, do SR e demais integrantes das geotecnologias. 


\section{TRABALHOS CONCERNENTES AO SR APRESENTADOS NO SIMPÓSIO BRASILEIRO DE GEOGRAFIA FÍSICA APLICADA}

No contexto da análise dos trabalhos concernentes ao SR em aplicações geográficas, ampliou-se o leque de eventos, e procedeu-se com a avaliação dos trabalhos publicados no XVI Simpósio Brasileiro de Geografia Física Aplicada. Este evento ocorreu no ano de 2015, na cidade de Teresina, estado do Piauí. Neste evento, contabilizou-se 75 (setenta e cinco) trabalhos que utilizaram de alguma ferramenta ou técnica de SR. A análise dos trabalhos submetidos ao XVI SBGFA considerou os seguintes itens: i) o número de trabalhos por eixo temático; ii) presença de metodologias coerentes ou inovadoras; iii) presença de referências internacionais; iv) a pertinência da metodologia empregada e a sua descrição e; v) emprego correto dos conceitos.

Os trabalhos analisados estavam distribuídos nos seguintes eixos temáticos:

1. Dinâmica, potencialidade e vulnerabilidades do Nordeste Brasileiro;

2. Análise Climática: métodos e técnicas, impactos e riscos;

3. Cartografia e geotecnologias para fins de planejamento e gestão do território;

4. Dinâmica da paisagem, recursos naturais e planejamento ambiental em áreas rurais e urbanas;

5. Geomorfologia e solos: epistemologia, técnicas, processos dinâmicos e mudanças na paisagem;

6. Bacias hidrográficas: métodos e técnicas de estudo, usos, ocupação e conflitos no Espaço Geográfico;

7. Geografia física: Ensino, pesquisa e extensão;

8. Biogeografia: Propostas teóricas, metodológicas e técnicas para fins de conservação ambiental;

9. Geoconservação, Geoturismo, Patrimônio geomorfológico e impactos ambientais.

No eixo temático "Dinâmica, potencialidades e vulnerabilidades do Nordeste Brasileiro", um total de 04 trabalhos utilizaram do SR. Todos os trabalhos apresentavam as metodologias comumente empregadas nesse tipo de pesquisa. O número de referências internacionais e nos trabalhos foi reduzido, sendo que apenas um trabalho apresentou 03 citações internacionais. Dos trabalhos analisados, notou-se que apenas um apresentava detalhadamente a descrição da metodologia empregada concernente ao SR. Em termos conceituais, excetuando-se um trabalho, os demais não apresentavam conceituações, ou quando presentes, estavam empregados de forma equivocada, como por exemplo, confundindo SR com Cartografia. 
No eixo temático "Análise Climática - métodos e técnicas, impactos e riscos", observou-se a presença de 03 trabalhos que fizeram o uso do SR. Os trabalhos aplicavam de metodologias já consolidadas referentes aos temas. Apenas um trabalho não apresentou referências internacionais. A maioria dos trabalhos apresentavam uma boa descrição das metodologias e o seu correto emprego. Em relação aos conceitos, observaram-se equívocos.

O eixo temático "Cartografia e geotecnologias para fins de planejamento e gestão do território", contou com a submissão de 31 trabalhos. Observou-se que mais de 90\% dos trabalhos submetidos ao eixo apresentavam metodologias já consolidadas e que estavam implementadas nos aplicativos. Em relação a presença de referências internacionais, não houve a citação de trabalhos em língua estrangeira referentes ao SR. Também notou-se a ausência da descrição pormenorizada da metodologia empregada.

No eixo temático intitulado "Geomorfologia e solos - epistemologia, técnicas, processos dinâmicos e mudanças na paisagem", notou-se a presença de 09 trabalhos que se pautaram no uso de ferramentas e técnicas de SR. Além das metodologias comumente empregadas, dois trabalhos realizaram procedimentos que envolviam a fusão híbrida de imagens orbitais e o mapeamento digital de solos. Notou-se a maior presença de referências em língua estrangeira. Do total de trabalhos, 06 apresentavam referências em língua estrangeira referentes ao SR. Contudo, apenas 03 trabalhos apresentavam a descrição metodológica detalhada.

O eixo temático "Bacias hidrográficas - métodos e técnicas de estudo, usos, ocupação e conflitos no Espaço Geográfico", contou com a submissão e 18 trabalhos que envolviam o uso do SR. Desses, a maior parte pautava na utilização de metodologias comumente empregadas. O número de trabalhos com citações em língua estrangeira de trabalhos de SR também foi baixo, totalizando 07. A maior parte dos trabalhos não apresentava detalhadamente a descrição dos procedimentos metodológicos.

No eixo temático "Geografia física - ensino, pesquisa e extensão", houve a submissão de 03 trabalhos. Não se observou citação de referências em língua estrangeira referente ao SR. Apenas um dos trabalhos apresentava a descrição pormenorizada da metodologia, especialmente, referente ao SR. O eixo temático "Biogeografia - Propostas teóricas, metodológicas e técnicas para fins de conservação ambiental", também apresentou 03 trabalhos que envolviam SR. As metodologias utilizadas foram as já consolidadas no meio acadêmico, e todos os trabalhos apresentavam pelo menos uma citação em língua estrangeira referente ao SR. Contudo, a descrição metodológica dos trabalhos eram resumidas. Por fim, o eixo temático "Geoconservação, Geoturismo, Patrimônio geomorfológico e impactos ambientais", apresentou apenas 01 trabalho que envolveu o 
uso do SR. O trabalho não apresentava referências estrangeiras sobre SR e a descrição metodológica era pouco detalhada.

De modo geral, os temas predominantes observados nos trabalhos submetidos ao XVI SBGFA foram: i) uso da terra e cobertura vegetal, ii) índices espectrais (predomínio de NDVI) e, iii) mapeamento geomorfológico. No contexto metodológico, observou-se que os trabalhos não apresentavam inovação metodológica. Comumente utilizam das metodologias já consolidadas e/ou implementadas nos aplicativos, variando apenas as áreas de estudo. Deve-se considerar que, por se tratar de um evento, os autores podem enviar trabalhos menos complexos, reservando os resultados finais para publicações em periódicos. Em relação a citação de literatura em língua estrangeira, nota-se o predomínio de citação de trabalhos nacionais.

Sobre a metodologia empregada nos trabalhos submetidos ao XVI SBGFA, constatouse a ausência de detalhamento dos procedimentos sobre o processamento digital de imagens. Especificamente, a comumente falta de correções atmosféricas em aplicações que a demandariam, como por exemplo, em trabalhos envolvendo índices espectrais. Outro descuido refere-se à ausência da descrição da etapa de validação estatística dos mapeamentos realizados. Foram identificados alguns equívocos concernentes aos conceitos dos elementos que integram as geotecnologias. Na grande parte dos trabalhos, observouse que, ou não há a definição do conceito, ou quando existe, está equivocado, como por exemplo, confundindo SR com Cartografia, SIG e/ou Geoprocessamento. Novamente, verificou-se a ausência de trabalhos abordando temas com o Lidar, radar, Vants, drones, dentre outros. Esta conjuntura pode ser reflexo de uma série de motivos, como: carência de recursos para aquisição de dados, falta de conhecimento teórico e prático na utilização de determinadas tecnologias, ausência de interesse em submeter trabalhos mais complexos no evento analisado, etc. Nota-se mais uma vez que, ao se ausentar dessas abordagens, o geógrafo perde também profissionalmente, comparando-se com outros profissionais, que muitas vezes não tem o domínio da abordagem espacial.

\section{CONSIDERAÇÕES FINAIS}

As discussões realizadas durante a realização do GT e a posterior análise dos trabalhos, incluindo os apresentados no SBGFA, evidenciam um panorama geral da aplicação do SR na geografia. É fundamental salientar que, o intuito deste trabalho e do GT não é o de tecer críticas aos trabalhos apresentados, muito menos, o de apenas polemizar. Tem sim, 
o objetivo de promover uma reflexão na comunidade geográfica que utiliza do SR, a fim de discutir sobre os pontos positivos e negativos dessa importante ferramenta que, no entendimento desses autores, cada vez mais deve ser utilizada pelo geógrafo. Embora para muitos, as ponderações deste trabalho possam ser interpretadas como ousadas, os autores apenas relatam o que foi observado no GT e nos trabalhos submetidos ao SBGFA, referentes ao tema SR. Os autores também têm ciência de que, o número reduzido de trabalhos submetidos ao GT no ENANPEGE deve-se em partes, ao fato de que ainda não é tradicional as discussões envolvendo geotecnologias nesse evento, especialmente, de SR. Haja visto que o GT proposto ocorreu pela primeira vez no ano de 2015. Adicionalmente, é sabido que na geografia há pesquisadores e grupos de pesquisas que aplicam o SR como, 'pesquisa pura', e cujos trabalhos, são direcionados a outros eventos, especialmente, o Simpósio Brasileiro de Sensoriamento Remoto (SBSR) e o Congresso Brasileiro de Cartografia (CBC). Estes dois eventos, principalmente o SBSR atuam como a vanguarda do SR, onde o estado da arte e as pesquisas mais complexas e inovadores de SR são ali apresentadas. Adicionalmente, nota-se um número expressivo de publicações de geógrafos indexadas nos periódicos internacionalmente respeitados.

De modo geral, os trabalhos submetidos ao GT proposto no ENANPEGE e, no SBGFA são aqueles onde o SR é uma ferramenta para geração de informação inicial, as quais serão utilizadas para análises mais complexas. Por exemplo, para posterior integração com dados de forma de relevo, de feições geomorfológicas, de estrutura urbana, de mobilidade, de questões fundiárias, de clima, de pedologia, dentre outros. Esta situação é observada nos trabalhos submetidos ao SBGFA, onde nove eixos temáticos apresentaram algum trabalho que utilizou do SR. Acreditamos que o número de trabalhos submetidos aos eventos que utilizam do SR e das demais geotecnologias deva aumentar gradativamente, até em função da inserção dessas tecnologias na geografia brasileira. Resta saber se em termos de qualidade metodológica, haverá avanços.

Os exemplos comuns de aplicação do SR na geografia são aqueles que envolvem o mapeamento de alguma variável de uso e cobertura da terra. Comumente estes trabalhos foram desenvolvidos com imagens de média resolução espacial (as disponibilizadas gratuitamente), e a partir do uso de classificadores implementados nos aplicativos de processamento digital de imagens. No contexto metodológico, parte dos trabalhos analisados possuía arcabouço metodológico consistente, porém, observaram-se muitos casos de inconsistências. Essa condição reduz a confiabilidade dos resultados alcançados. Um dos equívocos recorrentes foi o de trabalhos que envolviam a obtenção de índices espectrais como o NDVI, sem a execução apropriada de procedimentos de correção 
atmosférica. Notou-se também que, parte expressiva dos trabalhos faz o uso de sensores remotos de média resolução espacial. Contudo, algumas aplicações careciam de imagens de melhor resolução espacial. Dessa forma, o objetivo do trabalho não é alcançado em plenitude em função da natural incapacidade do produto utilizado, e os resultados tornamse discutíveis. Também se observou a baixa execução do procedimento de validação dos resultados alcançados, especialmente os que envolviam o mapeamento. Os resultados comumente eram descritos qualitativamente, sem validação estatística.

O SR, bem como as geotecnologias é uma importante ferramenta que aliada a rica formação do geógrafo, pode ser utilizada para diversas finalidades. As habilidades do geógrafo em analisar e refletir sobre os fatos e fenômenos que ocorrem no espaço geográfico podem ser potencializados com o uso integrado do SR e demais geotecnologias. Neste contexto, estes autores ousadamente expõem a necessidade de rever a forma como é conduzida o ensino de geotecnologias nos cursos de geografia. É comum verificar nas apresentações dos trabalhos o relato dos alunos, tanto de graduação como de pósgraduação, sobre a dificuldade de manipular os dados de SR e demais geotecnologias. Por exemplo, relatos que expõe que parte do tempo de um mestrado, por exemplo, é destinada a aprendizagem da parte prática concernente ao SR e geotecnologias.

Diante disso, a reflexão e a realização de pesquisas complexas e completas ficam limitadas. Adicionalmente, as discussões sobre os resultados alcançados podem ficar empobrecidas, em função não só da insuficiente carga teórica e prática de SR e demais geotecnologias, como também pelos outros temas geográficos contemplados na pesquisa. Embora este trabalho não tenha analisado as discussões presentes nos trabalhos, Machado (2014) em publicação que apresenta os enfoques e orientação metodológica de trabalhos apresentados na XI Conferencia Iberoamericana de sistemas de Informacíon Geográfica, nota a ausência na maioria dos trabalhos de uma "inter-relação de processos e fenômenos naturais, ambientais e sociais por meio de mapas temáticos analíticos de fatores ou de causa/ efeito". Parte desta dificuldade pode ser entendida em alguns casos, pela ausência de uma efetiva formação teórica e prática de SR e geotecnologias durante o curso de graduação. A formação mais consistente, tanto de teoria como de prática das geotecnologias pode conduzir a execução de trabalhos mais complexos, e cujos resultados possam ser melhor explorados. Adicionalmente, em termos profissionais, a formação sólida nesta área do conhecimento garantirá a atuação satisfatória do geógrafo em diferentes frentes de atuação.

Existe um amplo espaço de crescimento do SR e demais geotecnologias na Geografia. Contudo, o seu sucesso depende não só de melhor formação, mas também de mudanças estruturais na ciência geográfica. Diante disso, compactua-se da interpretação de Machado 
(2014), quando o autor aborda os "novos enfoques da geografia com o apoio das tecnologias da informação geográfica". O autor supracitado sugere que o desenvolvimento recente das Tecnologias da Informação Geográfica indica que está acontecendo uma mudança paradigmática que levará a novos fundamentos teóricos, conceituais e ontológicos para a consolidação da Ciência da Informação Geográfica. Assim, ousamos incluir o SR e as demais geotecnologias. Espera-se que realmente esteja ocorrendo a mudança sugerida por Machado (2014), o qual indica o beneficiamento de todas as expressões do conhecimento geográfico, independente da ênfase nos aspectos físicos ou humanos.

\section{REFERÊNCIAS}

1. BOYD, D. S. Remote sensing in physical geography: a twenty-first-century perspective. Progress in Physical Geography, v. 33, n. 4, p. 451-456, 2009.

2. ESTES, J. E.; JENSEN, J. R.; SIMONETT, D. S. Impacts of Remote Sensing on U.S. Geography. Remote Sensing of Environment, v. 10, p. 43-90, 1980.

3. FLORENZANO, T. G. Geotecnologias na geografia aplicada: difusão e acesso. Revista do Departamento de Geografia - USP, v. 17, p. 24-29, 2005.

4. FLORENZANO, T. G. Geomorfologia: conceitos e tecnologias atuais. (Org). São Paulo: Oficina de Textos, 2008.

5. FREITAS, M. I. C. Da cartografia analógica à neocartografia: nossos mapas nunca mais serão os mesmos? Revista do Departamento de Geografia - USP, volume especial Cartogeo, p. 23-39, 2014.

6. GEWIN, V. Mapping opportunities. Nature, v. 427, n. 6972, p. 376-7, 22 jan. 2004.

7. JENSEN, J. Introductory digital image processing: a remote sensing perspective. 3 ed. Upper Saddle River: Pearson Prentice Hall, 2005. 526p.

8. JENSEN, J. R. Sensoriamento Remoto do Ambiente. Tradução: EPIPHANIO, J. C. N.(coordenador)...[et al.]. São José dos Campos- SP: Editora Parêntese, 2009. 598 p.

9. LONGLEY, P. A.; GOODCHILD, M. F.; MAGUIRE, D. J.; RHIN, D. W. Sistemas e Ciência da Informação Geográfica. Tradução: SCHNEIDER, A. (coordenador) ...[et al]. Porto Alegre: Bookman, 2013.

10. MACHADO, R. P. P. Os novos enfoques da geografia como apoio das tecnologias da informação geográfica. Revista do Departamento de Geografia - USP, volume especial Cartogeo, p. 203$241,2014$.

11. MATTER, P. M. Computer processing of remotely sensed image: an introduction. 2 ed. West Sussex-England: John Wiley \& Sons, 1999, 292 p.

12. MONTANHER, O. C.; Souza Filho, E. E. Considerações sobre as formas de abordagem de imagens multiespectrais em geografia. Boletim de Geografia. v. 29, n. 2, p. 135-145, 2011. 
13. MONTANHER, O. C. Padrões espaço-temporais do transporte de sedimentos suspensos dos rios Amazônicos de águas brancas e estudo das relações com o clima e mudanças na cobertura do solo. 2016. 305 p. Tese (Doutorado). Universidade Estadual de Maringá - UEM, Programa de Pós-Graduação em Geografia, Maring:UEM, 2016.

14. NOVO, E. M. L. M. Sensoriamento remoto: princípios e aplicações. São Paulo: Editora Edgard Blücher, 1988.

15. PONZONI, F. J.; SHIMABUKURO, Y. E.; KUPLICH, T. M. Sensoriamento remote da vegetação. 2 ed. São Paulo: Oficina de Textos, 2012.

16. ROSA, R. Revista do Departamento de Geografia - USP, v. 16, p. 81-90, 2005.

17. SIMONETTI, D. S.; ULABY, F. T. (EDS). Manual of Remote Sensing. 2. ed. Vol. I. Falls Church VA: American Society of Photogrammetry, 1983, 1232 p.

18. XINGFA, G.; GUOLIANG, T.; XIAOWEN, L.; JIANNING, G. The quantification of remote sensing. Science in China Ser. E Engineering and Materials Science, v. 48, p. 1-11, 2005.

Artigo recebido em 30 de março de 2016.

Artigo aceito em 30 de abril de 2016. 